\title{
Exploration and learning of topographical relationships by the rat
}

\author{
TAKIJI YAMAMOTO \\ Hiroshima University, Hiroshima, Japan \\ SEYMOUR WAPNER and DAVID A. STEVENS \\ Clark University, Worcester, Massachusetts 01610
}

\begin{abstract}
In three experiments, the exploratory behavior of thirsty rats systematically progressed to areas distant from the starting location in an unfamiliar environment (table with seven objects on it). In a fourth experiment, this systematic pattern was disrupted when one of the objects in the new environment was familiar. Learning of spatial relations occurred during exploration, as evidenced by exploratory behavior on a test in which a previously neutral object in the environment became a source of water. Exploratory activity differed when the starting location was fixed in one corner of the table from when it was varied daily from corner to corner on a large table in one experiment; it did not differ with a table half that size in another experiment. The findings are taken to indicate that the development of the spatial structure consists of utilizing a known part of a given locale as anchor point or "base of operations" upon which is built a network of topographic relations among objects in particular locales.
\end{abstract}

While the extensity of the literature on exploratory behavior in the rat (see reviews by Barnett, 1958; Berlyne, 1960; Eibl-Eibesfeldt, 1970; Hinde, 1970) might suggest that it has been fully investigated, only a few workers have accumulated data pertinent to the proposition that the rat exhibits systematic exploration and learning of topographical relations among objects upon exposure to new environments. All of the pertinent variables have not yet been studied. For example, Dembo's classic study reported by Buytendijk (1931) introduced a large variety of objects in an open field but did not assess whether exploration was systematic; Hosoda (1971), who determined that exploratory behavior was systematic over time, did not use objects in the experimental area. Accordingly, it seemed worthwhile to conduct studies that would cover some of the features introduced by both of these investigators and also incorporate other variables that permit assessment of the course of exploratory behavior and topographical learning.

To study the learning of topographical relationships, four experiments were conducted. In the first, rats were exposed to a novel environment-a tabletop with a variety of objects on it-which they were free to explore. Then a rearrangement test was given, in which some objects were removed and/or changed in location for half of the subjects and left unchanged for the remaining subjects. Finally, a "reasoning" test (Maier, 1929) was conducted. For this purpose, a water fount was attached to one of the objects. The subjects were placed at the fount and allowed to drink from it. Then the subjects were placed elsewhere, and the route taken to the fount was recorded and timed.

The second experiment was similar to the first, but

Experiments 1, 2, and 4 were supported in part by grants from USPHS (MH-00348) and the Japanese Educational Ministry. Requests for reprints should be sent to Seymour Wapner, Heinz Werner Institute of Developmental Psychology, Clark University. refined procedures and controls were used and there was a reduction in the number of daily sessions and the time of each. This experiment also examined the effects of fixed vs. varied starting locations.

The third experiment further examined the effects of fixed vs. varied starting locations, but it used a table twice the size of that used in Experiment 2.

The fourth experiment was identical to Experiment 2, with the addition that it examined the effect of familiarity with one of the objects in the otherwise novel environment. Familiarity was achieved by placing that object in the rat's home cage prior to the free exploration sessions.

\section{EXPERIMENTS 1, 2, AND 3}

\section{Method}

Subjects. The subjects for Experiments $1(\mathrm{~N}=16)$ and 2 $(\mathrm{N}=32)$ were male rats of the Charles River CD strain. For Experiment 3, the subjects were 32 male rats of the Wistar strain. The subjects ranged in age from 56 to 71 days of age at the beginning of the experiments. All were housed singly and maintained on a 23.83-h water-deprivation schedule with a normal 12-h illumination cycle.

Apparatus. For Experiments 1 and 2, the rats were tested on a table top measuring $90 \times 180 \mathrm{~cm}$, covered with glass plates over which was laid a single piece of white paper with a black-line grid dividing the surface into $7215-\mathrm{cm}$ squares. Seven objects were located on the table according to the plan shown in Figure $1 \mathrm{~A}$. The objects were a pair of $10 \times 20 \times 9 \mathrm{~cm}$ hardware cloth ramps with wooden sides (Objects $\mathrm{A}$ and $\mathrm{E}$ ), a pair of inverted black plastic ash trays $10 \mathrm{~cm}$ in diameter (Objects $B$ and $F$ ), a pair of running wheels $18 \mathrm{~cm}$ in diameter (cylinders $10 \mathrm{~cm}$ in diameter in Experiments 2, 3, and 4) (Objects $C$ and $G$ ), and a piece of $22 \times 18 \mathrm{~cm}$ (reduced in height to $15 \mathrm{~cm}$ in Experiments 2, 3, and 4) hardware cloth mounted vertically on a hardware cloth base (Object D).

The level of illumination at the surface of the table was $377 \mathrm{~lx}$. Homogeneous white walls surrounded the table at a distance of $61 \mathrm{~cm}$ on three sides. The observer was located at one of the long sides of the table when observing and tracing the path of the rat. 

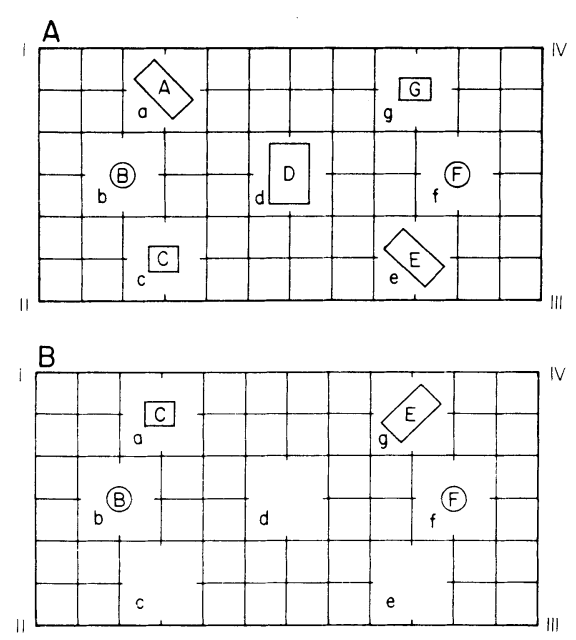

Figure 1. Plan of open field (A) with objects in original position and (B) after rearrangement: I-IV represent starting locations; A-G represent objects; a-g represent locales.

A video camera, which recorded the movement of the animal over the surface of the table as well as the elapsed time throughout the trial, was located directly above the table at a height of $210 \mathrm{~cm}$.

The refinements in apparatus in Experiments 2 and 3 included the use of recorded noise to balance that produced by the video camera, a one-way glass screen to hide the experimenter, and remote-controlled containers from which rats were released at the corners of the table.

In Experiment 3, the size of the table was doubled to $180 \times 360 \mathrm{~cm}$, and the black-line grid divided the surface into 72 30-cm squares.

Procedure. Initially, each rat was assigned a clean set of objects and paper flooring that were used for it exclusively throughout the study. Individually, a rat was placed on the table at one of the corners and permitted to move around with no constraints for a period of $8 \mathrm{~min}$ for Experiment 1, $4 \mathrm{~min}$ for Experiment 2, and $6 \mathrm{~min}$ for Experiment 3. Video recordings were made of the animal's behavior, and, concurrently, the experimenter mapped the animal's ambulations.

After each trial, the animal was returned to its home cage and given water for $10 \mathrm{~min}$. These free exploration trials were carried out for 12 days for Experiments 1 and 3 and for 8 days for Experiment 2. Then, on the 13th (or 9th) day, the rearrangement test was given. The subjects were divided into two groups matched for activity and starting location. One group (control) was treated the same as previously. The second group (rearrangement) was tested under conditions of rearrangement and removal of objects, as shown in Figure 1B. On the 14th or 10th) day, the objects were returned to their original positions, and another free exploration session was administered, followed by the "reasoning test." For this test, a water bottle was fastened to the center object (D). The subject was placed at the bottle and permitted to drink for $1 \mathrm{~min}$. Then the subject was returned to its original starting position, and its behavior was recorded by video camera and by the experimenter until it reached the bottle and began drinking.

For Experiment 1, the location at which a trial was started remained constant for each subject, but subgroups were formed so that an equal number of subjects started at each of the four corners of the table. For the other experiments, half of the subjects were always started from the same location on each trial (fixed groups) and half were started from different locations from trial to trial (varied groups). To control for possible sequence effects, four subgroups were formed, each having different starting location sequences.

\section{Results}

General activity was measured by the frequency of squares entered. In general, the activity showed an increase and then a decrease over the daily sessions. ANOVAs showed that for each experiment there was a significant day effect [for Experiments 1, 2, and 3, the findings were, respectively: $\mathrm{F}(11,165)=4.44, \mathrm{p}<.001$; $\mathrm{F}(7,210)=2.20, \mathrm{p}<.05 ;$ and $\mathrm{F}(11,330)=2.83, \mathrm{p}<.01]$ and a significant quadratic trend $[\mathrm{F}(1,165)=9.21$, $\mathrm{p}<.001 ; \mathrm{F}(1,217)=6.05, \mathrm{p}<.001 ;$ and $\mathrm{F}(1,330)=$ $19.96, \mathrm{p}<.001$, respectively].

Groups having fixed and varied starting locations did not differ in general activity in Experiment 2 $[F(1,30)=.40]$, but they did differ in Experiment 3 with the larger table $[F(1,30)=4.20, p<.05]$. The varied location group consistently entered more squares than the fixed location group. Starting location did not interact with days in any of the experiments.

Spatial distribution of exploratory behavior was measured by the ratio of time spent in "far" locales (two object areas of four squares each far from the starting location; e.g., Locales e and $\mathrm{f}$ in Figure 1 for Starting Location I), as compared with time spent in "near" locales (two object areas close to the starting location; e.g., Locales $a$ and $b$ for Starting Location I) and far locales combined. This ratio is shown plotted over days separately for each experiment in Figure 2. Betweendays effects were similar for the three experiments; generally, a negatively accelerating function was found. For Experiments 1,2, and 3, the ANOVAs showed significant day effects $[\mathrm{F}(11,165)=8.90, \mathrm{p}<.001 ; \mathrm{F}(7,210)=$ $3.77, \mathrm{p}<.01 ; \mathrm{F}(11,330)=6.32, \mathrm{p}<.001$, respectively]

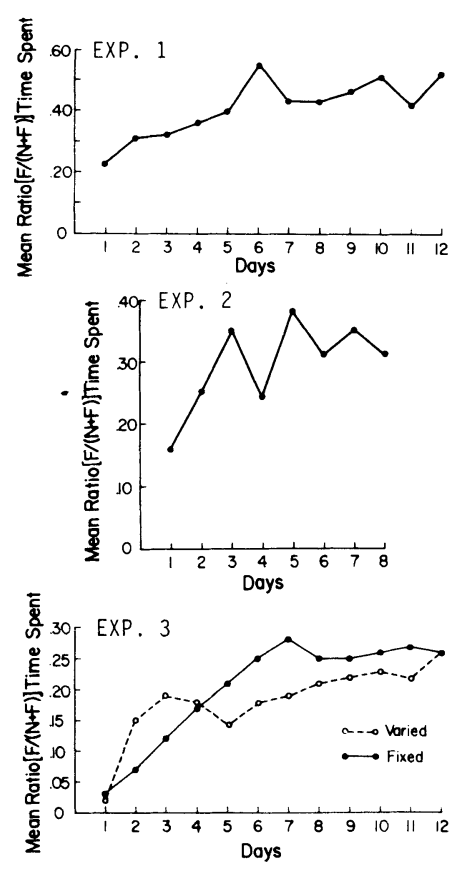

Figure 2. Spatial distribution of exploratory behavior for Experiments 1, 2, and 3. 
with significant linear and quadratic trends [Experiments $1-3$, respectively: $F_{1}(1,165)=62.94, F_{q}(1,165)=$ 12.50 , ps $<.001 ; \mathrm{F}_{1}(1,217)=11.22, \mathrm{~F}_{\mathrm{q}}(1,217)=5.13$, ps $<.05 ; F_{1}(1,330)=55.69, F_{q}(1,330)=10.15$, ps $<$ $.001]$.

A second measure of spatial distribution was used for Experiments 2 and 3. This was the frequency of rats entering a "far" locale at least once during a session. Fisher exact tests were used to compare the fixed and varied groups on each day. No differences were found for Experiment 2. In Experiment 3, the varied group showed a significantly $(\mathrm{p}<.02)$ higher frequency of animals entering the far locale than the fixed group on Days $5,6,7,11$, and 12 .

Object rearrangement and removal effects were measured by comparing the amount of time spent separately for locales having objects changed, objects removed, or objects unchanged during the rearrangement test session with that observed on the preceding day. The difference scores are presented in Table 1.

In locales with objects unchanged, there were no differences between the rearranged object and control groups in any of the five comparisons. Time spent in locales with objects removed (rearranged object groups) differed from time spent by control groups in four of the five comparisons. Similarly, time spent in locales where objects were changed differed from the controls in four of five instances.

For the reasoning test, the measure employed was the time the animal took to reach the water fount after it was released at the starting location. In all three experiments, the subjects that had the objects previously rearranged on the table took longer to go to the object holding the water fount than did the control subjects (objects not rearranged previously). For Experiment 1, the mean times to run to the fount for the rearranged object and control groups were 13.1 and $7.4 \mathrm{sec}$, respectively ( $\mathrm{p}=.06$ by a two-tailed Mann-Whitney test); for Experiment 2, the mean times for these groups were 21.0 and $9.7 \mathrm{sec}$, respectively $(\mathrm{p}<.02$, with fixed and varied location groups pooled for the analysis); and for Experiment 3, the mean times for these groups were
121.1 and $49.4 \sec (p<.02$, with fixed and varied groups pooled).

\section{EXPERIMENT 4}

Experiment 4 studied the effect of systematically introducing one object that differed from the others insofar as it was familiar to the rat. The specific aim of this experiment was to assess the effect of introducing a familiar object on exploratory behavior as a function of location (near, middle, and far from starting corner) of the familiar object.

\section{Method}

Subjects. The subjects were 24 experimentally naive male rats (Charles River CD), 65 to 81 days old. The housing conditions were as described for Experiment 1.

Apparatus. The apparatus and objects were the same as those used in Experiment 2; they were washed prior to reuse.

Procedure. The procedure followed was identical to that used in Experiment 2 except that (1) only fixed starting locations were used, (2) the rearrangement and reasoning tests were not employed, and (3) 10 days prior to Day 1 of testing, one of the objects used in testing served as the familiar object. This object was placed in the animal's home cage and remained there continuously, except when removed for use during testing. For eight animals, these objects were the ramps; for eight, they were the cylinders; and for eight others, they were the vertically mounted hardware cloth objects. For testing, the third object was located in Locale $d$ (the center of the table), and the first two objects were located either close to or far from the subject's starting location. For example, for Starting Location 1, the familiar object was located in Locale a (near), or it was located in Locale e (far). These locales are shown in Figure 1.

\section{Results}

The effect of introducing a familiar object on time spent in near and far locales was tested by varying the location of the familiar object (near, middle, or far locales) and comparing the time spent in near and far locales under two conditions: (1) with familiar object present (data from Experiment 4) and (2) with all objects novel (data from Experiment 2).

When the familiar object was in the near locale, its presence did not affect time spent in that locale (mean $=68.1 \mathrm{sec}$ with object present and mean $=72.0 \mathrm{sec}$

Table 1

Mean Difference Scores for Time Spent (in Seconds) in Original Object Locales and Tests of Significance (Mann-Whitney U) Between Object Rearrangement (R) and Control (C) Groups

\begin{tabular}{|c|c|c|c|c|c|c|c|c|c|}
\hline \multirow{2}{*}{$\begin{array}{l}\text { Starting } \\
\text { Position }\end{array}$} & \multicolumn{3}{|c|}{ Object Unchanged } & \multicolumn{3}{|c|}{ Object Removed } & \multicolumn{3}{|c|}{ Object Changed } \\
\hline & $\mathrm{R}$ & $\mathrm{C}$ & $\mathrm{U}$ & $\mathbf{R}$ & $\mathrm{C}$ & $\mathrm{U}$ & $\mathbf{R}$ & $\mathrm{C}$ & $\mathrm{U}$ \\
\hline & \multicolumn{9}{|c|}{ Experiment 1} \\
\hline \multirow[t]{2}{*}{ Fixed } & -.4 & 2.7 & 30 & -26.3 & 13.0 & $7 * *$ & 44.1 & -1.3 & $9 * *$ \\
\hline & \multicolumn{9}{|c|}{ Experiment 2} \\
\hline Fixed & -2.2 & 2.4 & 25 & .0 & 4.5 & 25 & 37.4 & -3.8 & $8^{*}$ \\
\hline \multirow[t]{2}{*}{ Varied } & 18.5 & 1.8 & 27 & -21.8 & -.5 & 12 & -19.5 & -7.2 & 30 \\
\hline & \multicolumn{9}{|c|}{ Experiment 3} \\
\hline Fixed & .1 & .8 & 35 & -1.3 & 9.9 & $5 \dagger$ & 26.2 & 8.6 & $8^{* *}$ \\
\hline Varied & 2.0 & 1.2 & 23 & -30.9 & -4.1 & $8^{* *}$ & 33.1 & 6.3 & $5 \dagger$ \\
\hline
\end{tabular}

Note-In Experiments 1 and 3, difference scores refer to Day 13 minus Day 12; in Experiment 2, they refer to Day 9 minus Day 8 . ${ }^{*} p<.05 . \quad{ }^{* *} p<.01 . \quad t p<.001$. 
without it). When the familiar object was in the middle locale, there was significantly less time spent in the near locale $($ mean $=28.4 \mathrm{sec})$ than when a familiar object was not present $($ mean $=72.0 \mathrm{sec})[\mathrm{F}(1,22)=4.50, \mathrm{p}<.05]$. Time spent in the far locale was not affected by the presence of the familiar object in the middle locale. When the familiar object was in a far locale, significantly more time was spent in the far locale (mean $=32.6 \mathrm{sec})$ than when there was no familiar object present (mean = $15.8 \mathrm{sec})[\mathrm{F}(1,22)=15.06, \mathrm{p}<.001]$, and significantly less time was spent in the near locale $($ mean $=61.1 \mathrm{sec})$ than when there was no familiar object present (mean = $72.4 \mathrm{sec})[\mathrm{F}(1,22)=5.06, \mathrm{p}<.05]$.

With one exception, there were no significant day effects or interactions of Presence of Familiar Object by Days. When a familiar object was in the near locale, there was a significant increase over days in time spent in the far locale $[F(7,154)=2.50, p<.05]$.

Thus, in general, placement of a familiar object affects exploratory behavior insofar as it makes for a relative increase in time spent in the far locale when the familiar object is placed there. Further, there is a decrease in the time spent in the near locale when the familiar object is placed in the middle locale.

\section{DISCUSSION}

The main findings were as follows: (1) Exploratory behavior unfolded systematically, with most exploration taking place closest to a starting location early in exposure to the new environment and progressing to distant areas after further exposure. (2) This systematic pattern was disrupted when one of the distant objects in the new environment was familiar to the rat. There was a displacement of exploratory activity toward the location of the familiar object. (3) Topographical relations among objects were learned during the course of exploration. This was shown by the effects of object rearrangement on (a) exploratory behavior and (b) speed of thirsty rats moving toward an object with a source of water (reasoning test). (4) Varying the starting location on general activity had significant effect on pattern of exploration with a large table but not with a smaller one.

The findings on systematic unfolding of exploratory behavior are congruent with our formulation that analyzes organismenvironment relationships from a holistic, developmental point of view (Wapner, Kaplan, \& Cohen, 1973). This approach assumes that the higher organism is goal directed and motivated to structure or put order into the environment and tends to orient itself in the environment and that the process of structuring and orienting in an environment unfolds in an orderly, systematic way. Moreover, it supports the "anchor-point hypothesis," which assumes that a salient location is employed as an anchor point or base of operations in relation to which locomotion and orientation takes place and in relation to which a cognitive organization of locales is established (cf. Schouela, Steinberg, Leveton, \& Wapner, in press).

These findings with rats are complemented by studies with infants and rhesus monkeys. In studying attachment and exploratory behavior in infants, Ainsworth and Witting (1969, p. 111) spoke of the child's "use of his mother as a secure base from which to explore the world." Rheingold and Eckerman (1969) refer to Ainsworth's (1963) characterization when describing their own findings on the infant's entry into a new environment. Similarly, Harlow and Zimmerman (1959, pp. 425 426) note that "after several sessions, the infants began to use the mother surrogate as a base of operations leaving her to explore and handle a stimulus and then returning to her before going to a new plaything." Rheingold and Eckerman's (1970) summary of the infant's behavior in a situation parallel to ours is pertinent; they state that "primarily by his own physical contacts with objects, near and distant, he learns the structural arrangements of objects in space and the tactual and other feedback properties of objects, both social and non-social" (p. 83). Whereas in that study the mother served such a function for the infant, in our situation the object at the start plays an analogous role in the development of the spatial organization.

\section{REFERENCES}

Ainsworth, M. D. The development of infant-mother interaction among the Ganda. In B. M. Foss (Ed.), Determinants of infant behavior (Vol. 2). London: Methuen, 1963.

Ainsworth, M. D., \& WitTing, B. A. Attachment and exploratory behavior of one-year-old children in a strange situation. In B. M. Foss (Ed.), Determinants of infant behavior (Vol. 4). London, Methuen, 1969.

BARnETT, S. A. Exploratory behavior. British Journal of Psychology, 1958, 49, 289-310.

Berlyne, D. E. Conflict, arousal, and curiosity. New York: McGraw-Hill, 1960.

BuytendiJk, F. J. J. Eine Methode zur Beobachtung von Ratten in Aufgabefreien situationen. Archives Neerlandaises de Physiologie de l'Homme et des Animaux, 1931, 16, 574-596.

EIBLE-EIBESFELDT, I. Ethology-The biology of behavior. New York: Holt, Rinehart, \& Winston, 1970.

Harlow, H. F., \& Zimmermann, R. R. Affectional responses in the infant monkey. Science, 1959, 130, 421-432.

Hinde, R. A. Animal behavior-A synthesis of ethology and comparative psychology (2nd ed.). New York: McGraw-Hill, 1970.

HosodA, K. Some aspects of open-field behavior in the rat. Bulletin of School of Education, Okayama University, 1971, 31, 45-71.

MAIE R, N. R. F. Reasoning in white rats. Comparative Psychology Monograph, 1929, 6(No. 29).

Rheingold, H. L., \& Eckerman, C. O. The infant's free entry into a new environment. Journal of Experimental Child Psychology, 1969, 8, 271-283.

Rheingold, H. L., \& Eckerman, C. O. The infant separates himself from the mother. Science, 1970, 168, 78-83.

Schouela, D. A., Steinberg, L. M., Leveton, L. B., \& W APNER, S. Development of the cognitive organization of an environment. Canadian Journal of Behavioral Science, in press.

Wapner, S., Kaplan, B., \& Cohen, S. B. An organismicdevelopmental perspective for understanding transactions of men in environments. Environment and Behavior, 1973, 5, 255-289.

(Received for publication November 19, 1979.) 